EPiC Series in Engineering
Volume 3, 2018, Pages 2359-2367
HIC 2018. 13th International
Conference on Hydroinformatics

\title{
High-Performance Integrated hydrodynamic Modelling of Storm Induced Floods at a Catchment Scale
}

\author{
Xilin Xia ${ }^{1,2}$, Qiuhua Liang ${ }^{1,2}$, Xiaodong Ming ${ }^{2}$ \\ ${ }^{1}$ School of Architecture, Building and Civil Engineering, Loughborough University, \\ Loughborough LE11 3RU, UK \\ ${ }^{2}$ School of Engineering, Newcastle University, Newcastle upon Tyne NE1 7RU, UK \\ x.xia2@lboro.ac.uk
}

\begin{abstract}
Flooding is one of the most common types of natural hazards. The current practice of large-scale fluvial flood modelling relies on the use of hydrological models to predict upstream discharge hydrograph to drive inundation modelling at downstream. However, the oversimplified representation of both catchment topographies and hydraulics make hydrological models heavily rely on model parameterisation and calibration. This makes the modelling strategy unsuitable for prediction of extreme events featured with highly transient hydraulic processes, for which high-quality hydrological data is commonly missing to support model parameterisation and calibration. In this paper, the HighPerformance Integrated hydrodynamic Modelling System (HiPIMS) has been adapted and applied to the whole $2500 \mathrm{~km}^{2}$ Eden catchment in the UK to reproduce the flood event caused by Storm Desmond in December, 2015. Without necessity of intensive calibration and using hydrographs as boundary conditions, satisfactory results have been obtained for both inundation extent and water level time series in channels, in comparison with observations. Accelerated by multiple modern graphic processing units (GPUs), the model runs more than 20 times faster than real time for the simulation of the whole catchment at $20 \mathrm{~m}$ resolution. The results successfully demonstrate HiPIMS as a promising tool for real-time flood forecasting and flood risk assessment.
\end{abstract}

\section{Introduction}

Flooding is one of the most common types of natural hazards. Each year, it accounts for one third of the total loss caused by all natural hazards combined according to the EM-DAT disaster database 
[CRED, 2018]. Recent studies [IPCC, 2014] suggested that climate change may bring more extreme weather events and cause more frequent and severe floods in the future.

The current practice of large-scale fluvial flood modelling relies on the use of hydrological models (e.g. [Ewen et al., 2000; Beven and Freer, 2001]) to predict upstream hydrograph to drive inundation modelling at downstream. However, applying hydrological models to simulate extreme events featured with transient processes faces several fundamental difficulties. The first difficulty comes from their oversimplified physical representation of terrain topographies and hydrological/hydrodynamic processes. Hydrological models use coarse grids to enable catchment-scale simulations, at the expense of losing finer details of topographies. Conceptual or simplified hydrodynamic models are used for flow routing on hillslopes and in channels ([Lighthill and Whitham, 1955; Cunge, 1969; Govindaraju, 1988]) . Therefore, important physical processes, such as backwater effects and transcritical flows, cannot be adequately represented by hydrological models, leading to heavy reliance on model parameterisation and calibration. Particularly for those severe flood events caused by intense rainfall, the catchment and floodplain usually undergo highly transient processes in both space and time. Parameterising and calibrating hydrological models in such situations would be very difficult, if not impossible, because of the models' intrinsic inability to appropriately represent the underline physical processes and the involvement of large set of parameters. Another difficulty comes from the lack of high-quality data to calibrate hydrological models. Normally, hydrological models are calibrated against outlet discharge hydrographs derived from rating curves representing the regression relationship between river stage and discharge. But rating curves are subject to great uncertainties for extreme events [Domeneghetti et al., 2012]. Under extreme flow conditions, necessary measurements for deriving rating curves are often scarce or unavailable. Therefore, the discharge hydrographs are often not genuine, but derived from extrapolation of the rating curves developed for non-extreme conditions.

Recent advances in high-performance computing technologies have provided a new prospect for large-scale flood modelling and can hopefully circumvent the aforementioned difficulties. Highperformance computing based on Graphic Processing Units (GPUs) may speed up a model for up to two orders ([Smith and Liang, 2013; LIANG et al., 2016]) and enable us to apply full 2D hydrodynamic models to support flood modelling over a whole catchment at a very high resolution to represent fine topographies and complex hydraulics. Full catchment-scale simulation can effectively avoid the use of discharge hydrographs as boundary conditions and the associated uncertainties of using rating curves.

In this study, the High-Performance Integrated Hydrodynamic Modelling System (HiPIMS) recently developed by the authors is adapted and applied to simulate a full-scale flood event, from the upstream rainfall-runoff to the downstream inundation, in the $2500 \mathrm{~km}^{2}$ Eden Catchment. The flood event was directly caused by Storm Desmond hitting the catchment and many other places in the UK in December 2015. Successful reproduction of this large-scale flood event demonstrates the advantages of using a full-scale hydrodynamic model for fluvial flood modelling.

\section{The model - HiPIMS}

HiPIMS solves the vectorised form of the 2D shallow water equations (SWEs) written as

$$
\frac{\partial \boldsymbol{q}}{\partial t}+\frac{\partial \boldsymbol{f}}{\partial x}+\frac{\partial \boldsymbol{g}}{\partial y}=\boldsymbol{S}_{b}+\boldsymbol{S}_{f}
$$


where $\boldsymbol{q}$ is the vectorised conserved flow variable, $\boldsymbol{f}$ and $\boldsymbol{g}$ are the the $x$-and $y$-direction flux vectors, and $\boldsymbol{S}_{b}$ and $\boldsymbol{S}_{f}$ are the bed slope and friction terms. These vector terms are given by

$$
\begin{aligned}
& \boldsymbol{q}=\left[\begin{array}{c}
h \\
h u \\
h v
\end{array}\right], \boldsymbol{f}=\left[\begin{array}{c}
u h \\
u^{2} h+\frac{1}{2} g h^{2} \\
u v h
\end{array}\right], \boldsymbol{g}=\left[\begin{array}{c}
v h \\
u v h \\
v^{2} h+\frac{1}{2} g h^{2}
\end{array}\right], \\
& \boldsymbol{S}_{b}=\left[\begin{array}{c}
0 \\
-g h \frac{\partial b}{\partial x} \\
-g h \frac{\partial b}{\partial y}
\end{array}\right], \boldsymbol{S}_{f}=\left[\begin{array}{c}
0 \\
-\frac{\tau_{b x}}{\rho} \\
-\frac{\tau_{b y}}{\rho}
\end{array}\right] .
\end{aligned}
$$

where $u$ and $v$ are the $x$ - and $y$-direction depth-averaged velocities, $\rho$ is the density of water, $h$ is the water depth, and $\tau_{b x}$ and $\tau_{b y}$ are the frictional stresses calculated using the Manning formula:

$$
\begin{aligned}
& \tau_{b x}=\rho C_{f} u \sqrt{u^{2}+v^{2}}, \quad \tau_{b y}=\rho C_{f} v \sqrt{u^{2}+v^{2}}, \\
& \text { with } \\
& C_{f}=\frac{g n^{2}}{h^{\frac{1}{3}}}
\end{aligned}
$$

in which $n$ is the Manning coefficient.

A Godunov-type finite volume method is used to solve the SWEs. The resulting time-marching scheme is given as

$$
\boldsymbol{q}^{n+1}=\boldsymbol{q}^{n}-\frac{\Delta t}{\Omega_{i}} \sum_{k=1}^{N} \boldsymbol{F}_{k}\left(\boldsymbol{q}^{n}\right) l_{k}+\Delta t\left(\boldsymbol{R}_{i}^{n}+\boldsymbol{S}_{b i}^{n}+\boldsymbol{S}_{f i}^{n+1}\right),
$$

in which subscripts $i$ and $k$ are the indices of a cell and its cell edges, superscript $n$ denotes the time level, $\boldsymbol{F}_{k}(\boldsymbol{q})$ is the flux normal to the cell boundary, $l_{k}$ is the length of cell edge $k, \Omega_{i}$ is the cell area, and $\Delta t$ is the time step.

The flux terms are calculated using the HLLC Riemann solver ([Toro, 2001]). HiPIMS has adopted the recently developed Surface Reconstruction Method (SRM) for discretising slope source terms and the fully implicit scheme for discretising friction source terms (see [Xia et al., 2017] for details).

To enable high-resolution catchment-scale simulating involving tens of millions of cells, HiPIMS has been developed through the combination of the Nvidia CUDA programming framework and C++ 11 multi-threading techniques to support multi-GPU accelerations. 


\section{The case study - Storm Desmond event in Eden catchment}

\subsection{Description of the Storm Desmond event}

In December 2015, an extratropical cyclone named Desmond hit the northern part of the UK, leading to severe floods in north-western England and Scottish borders. The 48 hours total rainfall ranged from $174 \mathrm{~mm}$ to $405 \mathrm{~mm}$, which is more than one third of the average annual rainfall in the UK. Carlisle, located at Eden River mouth, became one of the worst flooded cities at the time. During the event, the whole $2500 \mathrm{~km}^{2}$ of Eden Catchment was covered by intense rainfall, and the Eden River experienced a peak discharge over $1700 \mathrm{~m}^{3} / \mathrm{s}$, more than three times bigger than its normal flow rate.

\subsection{Data and model setup}

The simulation domain covers the whole Eden River catchment, for which a map is provided in Figure 1. Digital Elevation Model (DEM) at $20 \mathrm{~m}$ resolution is used to set up the model for the proposed full-scale flood simulation across the whole catchment, which is resampled from the $5 \mathrm{~m}$ DEM released by the UK Ordnance Survey. The simulation involves 6.25 million computational cells/nodes. The NIMROD $1 \mathrm{~km}$ rainfall radar data released by UK met office, dated from $5^{\text {th }}$ to $8^{\text {th }}$ December 2015 , is used as input to drive the simulation. The median and mean rainfall intensities extracted from the rainfall radar data is shown in Figure 2, indicating that most rainfall was in the period from $5^{\text {th }}$ to $8^{\text {th }}$ December 2015, but there was also a significant amount of rainfall before $5^{\text {th }}$ December 2015. Open boundary conditions are imposed at the domain boundaries to allow free outflow. It is worth emphasizing that there is no need to use any inflow hydrographs as boundary conditions because the whole catchment has been included in the simulation. During the simulation, channel flows are automatically generated through the rainfall-runoff and flow converging processes. Zero infiltration is assumed due to the antecedent rainfall events and fully saturated soil. Without considering infiltration, the model has effectively only one parameter, i.e. the Manning coefficient, to determine. For the current simulation, the Manning coefficient is chosen to be $0.025 \mathrm{sm}^{-1 / 3}$ for river channels and $0.075 \mathrm{sm}^{-1 / 3}$ for floodplains and hillslopes, as suggested in common hydraulics text books. 


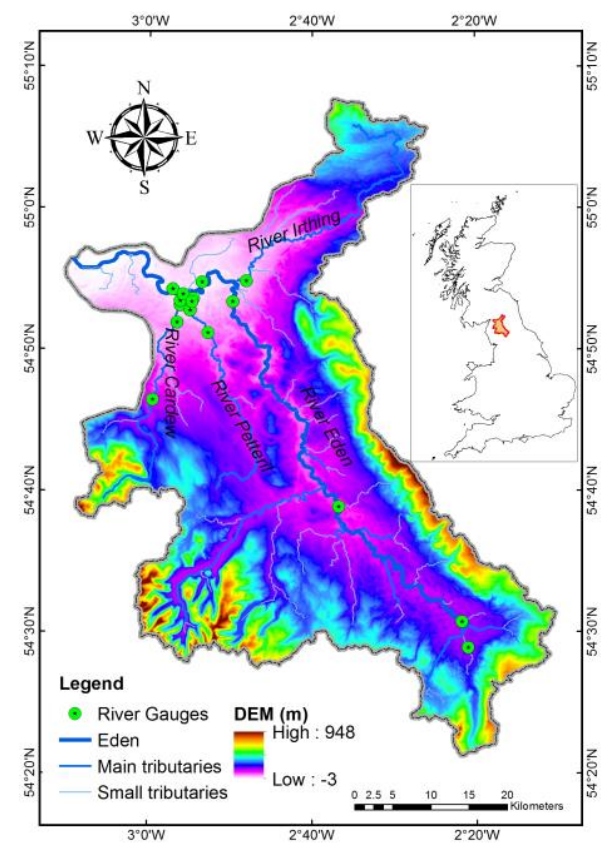

Figure 1 Map of the Eden catchment

For practical flood modelling, deciding the initial conditions to start a simulation is always an important but difficult step. In this study, antecedent rainfall data is used to 'warm up' the model. Two days of rainfall data before the event has been used to drive the simulation on the dry barren topographies to create continuous river flows. After the rainfall stops, the simulation continues for another ten hours to allow most of the runoff to converge to river channels and ensure the water depth at chosen gauges to be consistent with measurements. The outputs in terms of water depth and velocities are then used as the initial conditions for the simulation of Desmond floods.

There are several river gauges on the River Eden and its tributaries which record the water levels. The recorded water levels at four gauges on the River Eden and the post-event survey of the flood inundation extent provided by the Environment Agency are used to validate the simulation.

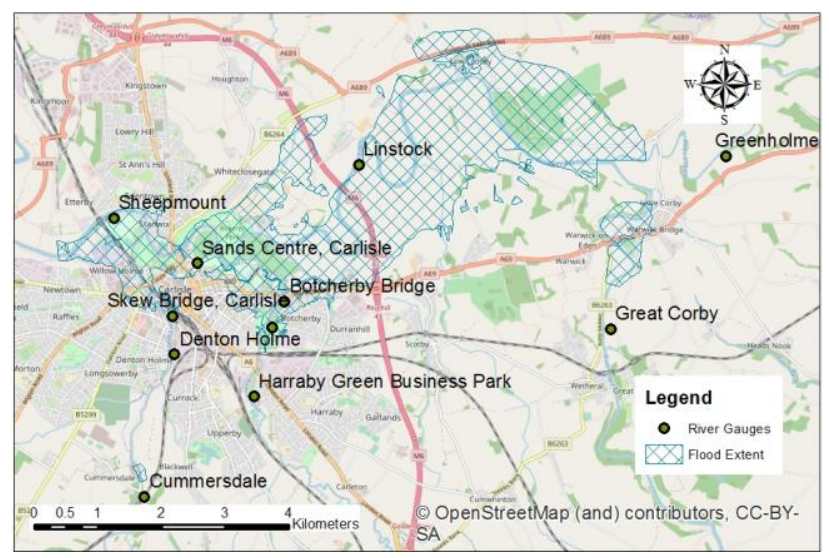


Figure 2 Post-event survey of the flood extent at Carlisle.

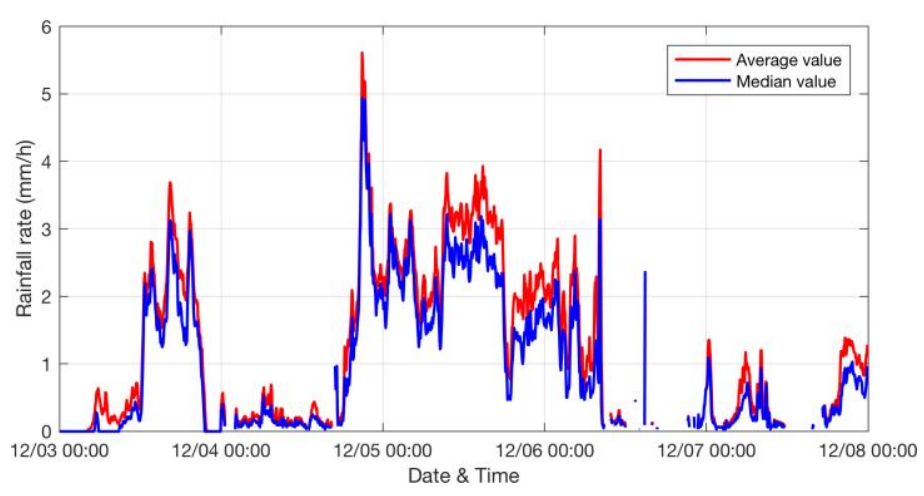

Figure 3 Average and median rainfall intensities over the whole Eden River catchment.

\section{Results and discussions}

The simulated inundation map of the downstream city, Carlisle, is compared with post-event survey provided the UK Environment Agency in Figure 4. The surveyed flood extent has been well predicted by HiPIMS. But certain flooded areas as predicted are not identified in the post-event survey map. Most of the discrepancies are found in the areas near the river gauges 'Great Corby' and 'Denton Holme'. The difference may be caused by two reasons: 1) the upstream region near 'Great Corby' was not surveyed and no information is available in terms of flood extent; 2) the downstream region between Skew Bridge and Denton Holme (bottom left corner in Figure 1) was not flooded during the event because it was well protected by the flood walls built after 2010, but the flood walls unfortunately cannot be well represented by the DEM at the $20 \mathrm{~m}$ resolution. To further validate the simulation results, the predicted time series of water level at three gauges along the Eden River are plotted and compared with measurements in Figure 5 and close agreement has been observed in all three gauges. The NashSutcliffe efficiency (NSE) has been adopted herein to quantitatively confirm the accuracy of the numerical prediction, which is defined as

$$
N S E=1-\frac{\sum_{1}^{N}\left(h_{m}^{n}-h_{o}^{n}\right)}{\sum_{1}^{N}\left(h_{m}^{n}-\bar{h}_{o}\right)}
$$

where $\mathrm{N}$ is the total number of time steps, $h_{o}^{n}$ is the observed water level at $\mathrm{t}=\mathrm{n}, h_{m}^{n}$ is the simulated water level at $\mathrm{t}=\mathrm{n}$, and $\bar{h}_{o}$ is the mean observed water level. $N S E=1$ suggests perfect agreement between the prediction and the observation. The NSEs for this simulation are listed in Table 1. All of the calculated NSEs are greater than 0.85, and the mean NSE for the four gauges on the Eden river is calculated to be 0.92 , indicating that the observed water levels have been successfully reproduced with high accuracy by the current model.

Without the necessity of intensive model calibration (using the Manning coefficients suggested in hydraulics text books), the simulation results are deemed to be satisfactory. The current hydrodynamic 
model HiPIMS is therefore considered to outperform conventional hydrological models due to its capability in predicting reliable results without intensive calibration. The model takes 2 hours 50 minutes to finish the simulation of this 72 hours highly dynamic event on a server equipped with two Tesla K80 and four Tesla K40 GPUs, more than 20 times faster than real time.

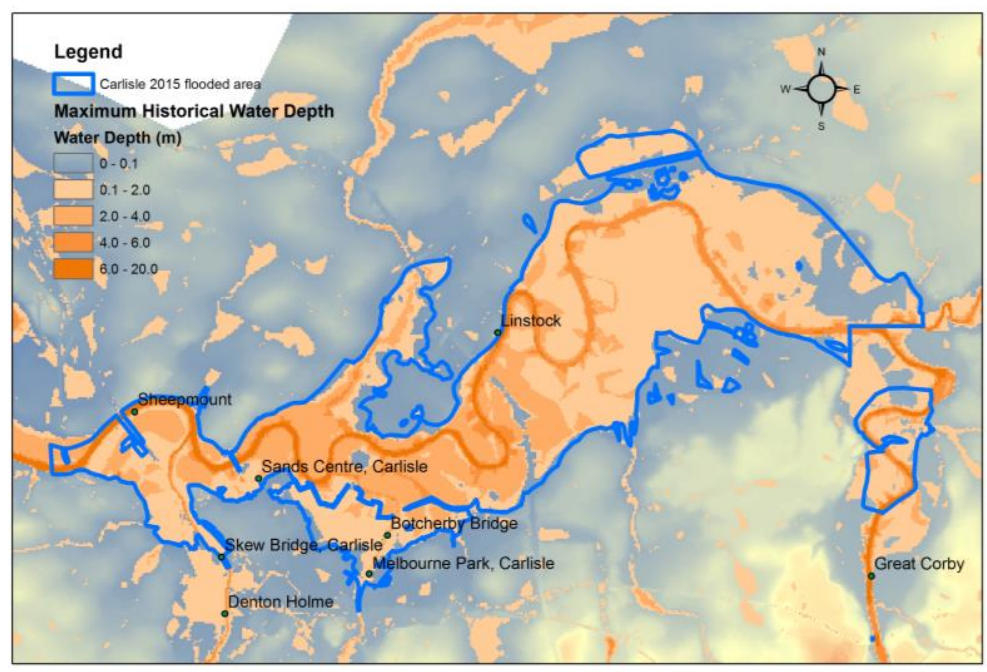

Figure 4 Simulated inundation map of Carlisle compared with the post-event survey.
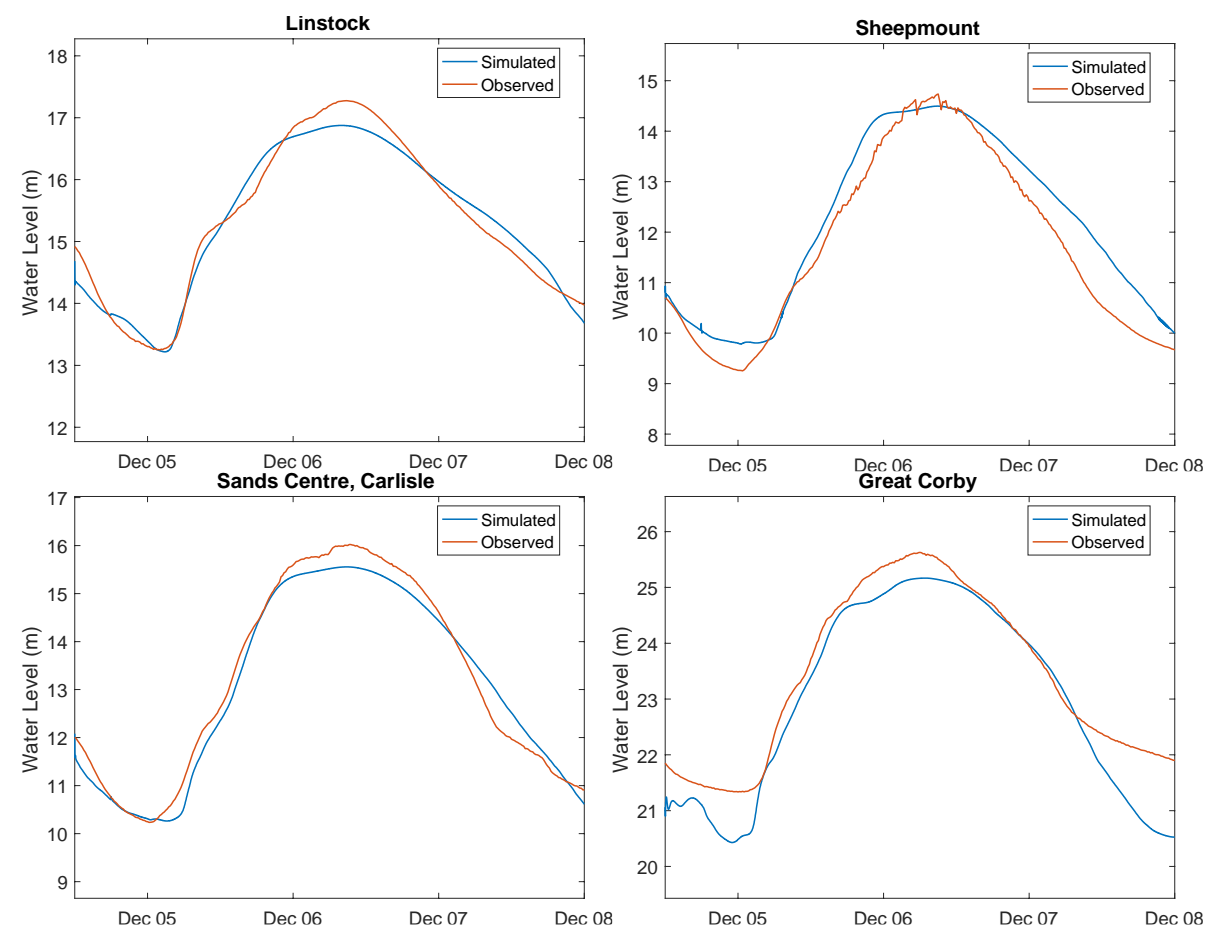

Figure 5 Simulated and measured water levels at three gauges along the Eden River. 


\begin{tabular}{ll}
\hline Gauge Names & NSE \\
\hline Linstock & 0.96 \\
Sheepmount & 0.90 \\
Sands Centre, Carlisle & 0.97 \\
Great Corby & 0.85 \\
Mean & 0.92 \\
\hline
\end{tabular}

Table 1 NSEs calculated against measured water levels at the four river gauges on the Eden River.

\section{Conclusions}

In this work, we have applied HiPIMS to simulate the flood event caused by Storm Desmond in the $2500 \mathrm{~km}^{2}$ Eden catchment. With the aid of multiple modern GPUs, HiPIMS is able to simulate the rainfall-runoff-flooding processes in the whole catchment from the upstream to the downstream using a $20 \mathrm{~m}$ uniform grid to represent the relatively fine details of the catchment topographies. Because the whole catchment has been included in the simulation domain, river flow hydrographs are not needed as boundary conditions to drive the model, which avoids the associated uncertainties of rating curves. The advantages of catchment-scale flood simulation using HiPIMS are evidenced by accurate prediction of both the inundation extent and water level hydrographs without intensive calibration. The model runs more than 20 times faster than real time on a multiple-GPU server. The superior accuracy and efficiency of HiPIMS show its great potential for a wide range of applications in flood risk assessment and realtime flood forecasting.

\section{Acknowledgment}

This work is funded by the NERC through projects SINATRA/TENDERLY (Grant No. NE/K008781/1) and Flood-PREPARED (Grant No. NE/P017134/1).

\section{References}

Beven, K., and J. Freer (2001), A dynamic TOPMODEL, Hydrol. Process., 15(10), 1993-2011, doi:10.1002/hyp.252.

CRED (2018), EM-DAT: The international disaster database, Available from: http://www.emdat.be

Cunge, J. A. (1969), On The Subject Of A Flood Propagation Computation Method (Musklngum Method), J. Hydraul. Res., 7(2), 205-230, doi:10.1080/00221686909500264.

Domeneghetti, A., A. Castellarin, and A. Brath (2012), Assessing rating-curve uncertainty and its effects on hydraulic model calibration, Hydrol. Earth Syst. Sci., 16(4), 1191-1202, doi:10.5194/hess16-1191-2012.

Ewen, J., G. Parkin, and P. E. O'Connell (2000), Shetran : Distributed River B Asin F Low Modeling System, J. Hydrol. Eng., 5(JULY), 250-258. 
Govindaraju, R. . (1988), On the diffusion wave model for overland flow: 1. Solution for steep slopes, Water Resour. Res., 24(5), 734-744.

IPCC (2014), Climate Change 2014: Impacts, Vulnerability and Adaptation.

LIANG, Q., L. SMITH, and X. XIA (2016), New prospects for computational hydraulics by leveraging high-performance heterogeneous computing techniques *, J. Hydrodyn., 28(6), 977-985, doi:10.1016/S1001-6058(16)60699-6.

Lighthill, M. ., and G. . Whitham (1955), On Kinematic Waves . I . Flood Movement in Long Rivers, Proc. R. Soc. A Math. Phys. Eng. Sci., 229(1178), 281-316.

Smith, L., and Q. Liang (2013), Towards a generalised GPU/CPU shallow-flow modelling tool, Comput. Fluids, 88, 17-20.

Toro, E. F. (2001), Shock-capturing methods for free-surface shallow flow, John Wiley \& Sons, Ltd.

Xia, X., Q. Liang, X. Ming, and J. Hou (2017), An efficient and stable hydrodynamic model with novel source term discretization schemes for overland flow and flood simulations, Water Resour. Res., doi:10.1002/2016WR020055. 